\title{
Fluid flow in mid- to deep crustal shear systems: Experimental constraints, observations on exhumed high fluid flux shear systems, and implications for seismogenic processes
}

\author{
Stephen F. Cox \\ Centre for Advanced Studies of Ore Systems, Department of Geology and Research School of Earth Sciences, \\ The Australian National University, Canberra, ACT 0200, Australia
}

(Received January 8, 2002; Revised August 9, 2002; Accepted August 26, 2002)

\begin{abstract}
The aseismic parts of shear systems at mid- to deep crustal levels can localise the supply of deeply-sourced, high pressure fluids into the shallower level parts of these systems in the seismogenic regime. Even during deformation at elevated temperatures in mid- to deep crustal shear zones, high pore fluid factors promote grainscale to macroscopic fracture growth and permeability enhancement. The evolution of permeability is governed by dynamic competition between crack growth and crack sealing/healing processes. Steady state creep below the seismic-aseismic transition leads to steady state permeability and continuous fluid flow. In contrast, within and near the base of the seismogenic regime, large cyclic changes in permeability can lead to episodic fluid flow and fluctuations in fluid pressure. At mid-crustal depths, temporal and spatial variations in pore fluid pressure and shear stress within shear networks influence rupture nucleation via cyclic changes in shear strength. Fluid pressure and shear stress cycling can also drive repeated transitions between interseismic creep and rapid, co-seismic slip. Reaction-weakening and reaction-strengthening, during hydrothermal alteration in fluid-active shear systems, can also drive transitions between seismic and aseismic behaviour on longer time-scales.
\end{abstract}

\section{Introduction}

Faults and shear zones exhumed from mid- to lower crustal depths commonly contain evidence indicating that they have localised the flow of fluids through the crust. Fluid migration through faults and shear zones may influence the shear strength of these structures via the effects of (1) chemical fluid-rock reaction on the mineralogical composition of the deforming rocks, and (2) by inducing changes in fluid pressure and thus, effective stress. Fluid-driven changes in shear strength in shear systems may be an important factor influencing earthquake nucleation and recurrence near the base of the seismogenic regime in the continental crust (Sibson, 1996).

This contribution firstly reviews experimental results to illustrate how deformation and fluid pressures can influence the permeability of actively deforming rocks at mid- to deep crustal levels. Evidence for the involvement of fluids in midto deep crustal shear systems is then illustrated, in particular, using examples of high fluid flux shear systems which host Archaean gold deposits in Western Australia. We highlight how feedbacks between deformation, fluid flow and fluidrock reaction may influence episodic rupture nucleation near the base of the continental seismogenic regime in fluid-active geodynamic environments.

Copy right(c) The Society of Geomagnetism and Earth, Planetary and Space Sciences (SGEPSS); The Seismological Society of Japan; The Volcanological Society of Japan; The Geodetic Society of Japan; The Japanese Society for Planetary Sciences.

\section{Relationships Between Deformation and Perme- ability}

\subsection{Deformation-enhanced permeability}

At high temperatures in isostatically stressed mineral-fluid systems, pore geometry is controlled largely by minimisation of interfacial surface energies. Experimental studies indicate that for many mineral-fluid systems, pore connectivity is lost at porosities less than a few percent (Holness, 1997). Accordingly, at mid- to deep crustal levels most rocks will be effectively impermeable unless deformation actively generates interconnected fracture networks.

The evolution of permeability in initially low permeability rocks during deformation involving both intragranular plastic deformation and grain-scale crack growth is illustrated by experimental studies on calcite rocks (Zhang et al., 1994). At low effective confining pressures, permeability increases with increasing strain can be very rapid and large (Figs. 1(a), (b)). For example, at an effective confining pressure of $30 \mathrm{MPa}$, permeability increases by two orders of magnitude within 3\% shortening, and increases by a further order of magnitude by $10 \%$ shortening. Only small increases in permeability occur with higher strains.

Major increase in permeability, at strains as low as a few percent, is associated with growth of microcracks and rapid development of connectivity in grain-scale crack networks. Such behaviour persists well into the dominantly crystal plastic deformation regime, provided that pore fluid factors (ratio of pore fluid pressure to vertical stress) are high enough to facilitate some deformation by microcracking (Fischer and Paterson, 1992; Stormont and Daemen, 1992; 

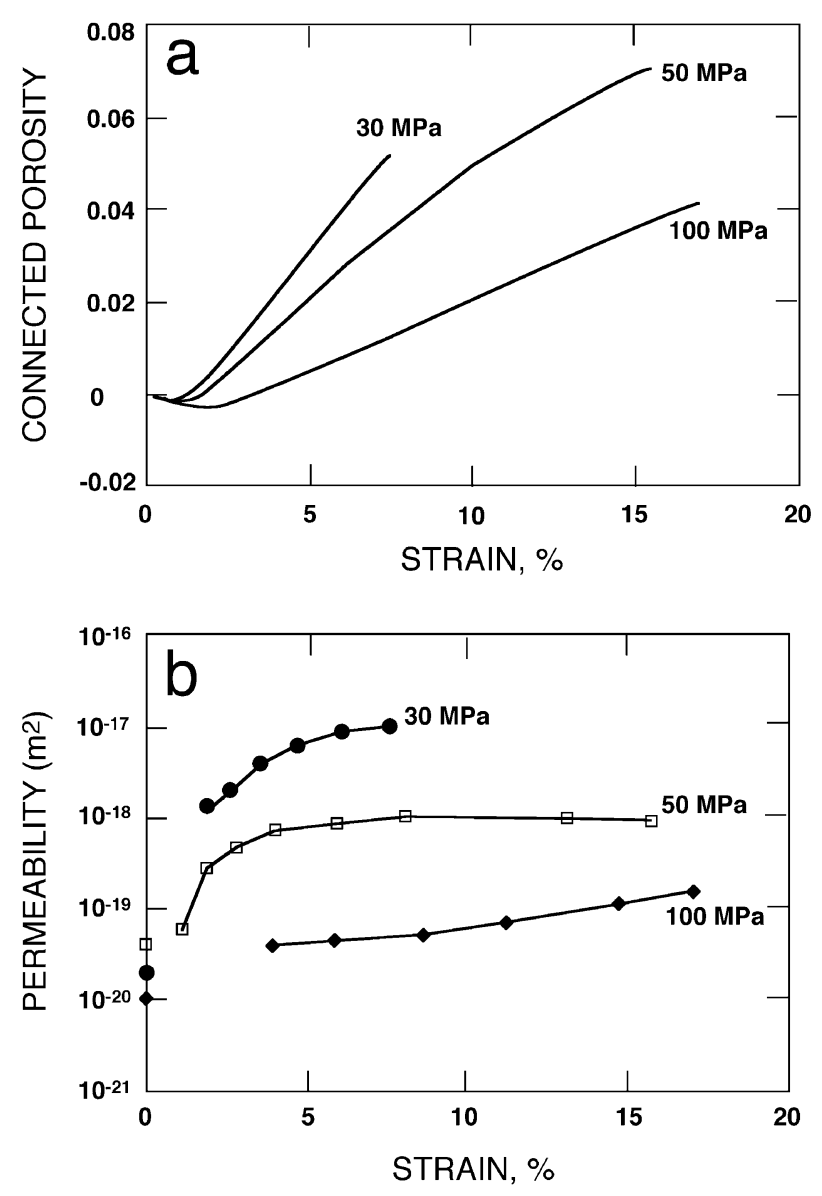

Fig. 1. Relationship between (a) porosity and strain, and (b) permeability and strain, as a function of effective confining pressure during deformation of Carrara marble at room temperature and in the presence of argon pore fluid. Relationships at effective confining pressures of $30 \mathrm{MPa}, 50$ $\mathrm{MPa}$ and $100 \mathrm{MPa}$ are illustrated. Confining pressure, $300 \mathrm{MPa}$; strain rate, $1.2 \times 10^{-4} \mathrm{~s}^{-1}$ (after Zhang et al., 1994).

Zhang et al., 1994; Peach and Spiers, 1996). With increase in both temperature and effective confining pressure, brittle/frictional processes are impeded and intracrystalline plasticity is favoured. So, crack growth rates reduce, and the critical strain required to develop a well-connected, high permeability crack network increases with increasing effective confining pressure.

The experimental studies demonstrate that where high fluid pressures produce low effective confining pressures, grain-scale crack growth significantly increases the permeability of active shear zones relative to their host-rocks, even though most displacement may be accommodated by microscopically ductile deformation mechanisms such as dislocation flow and dissolution-precipitation creep. A significant aspect of the experimental work is that fracture networks can develop high crack connectivity and high permeability at very low strains.

\subsection{Competition between permeability-enhancement} and permeability-reduction processes

At depth in the Earth's crust, especially at elevated temperatures in the presence of reactive pore fluids, healing and sealing of fractures can cause crack connectivity and permeability to decrease on time-scales that are short relative to the lifetimes of individual faults and shear zones. Accordingly,

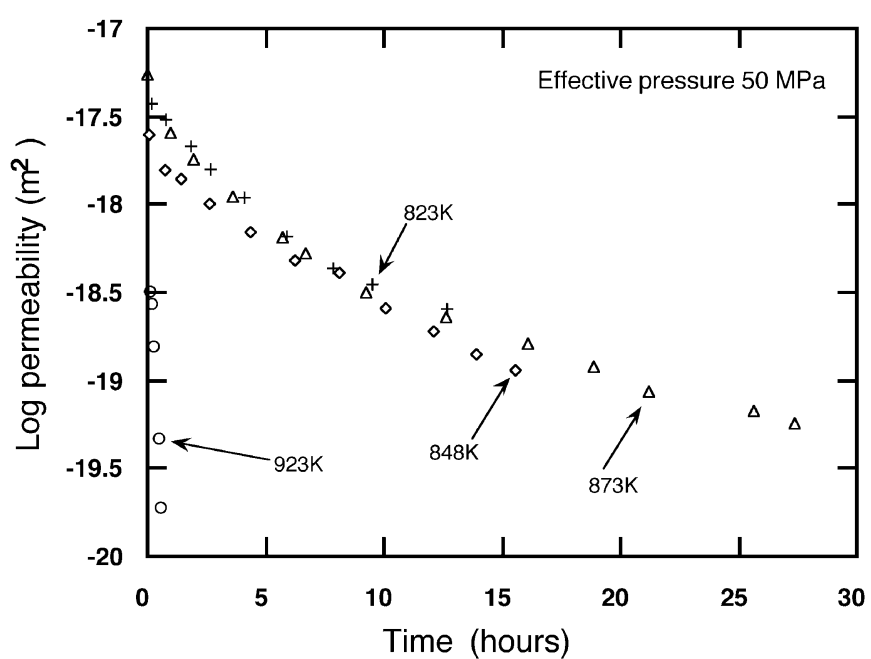

Fig. 2. Time-dependent changes in permeability associated with healing of grain-scale, deformation-induced microcracks in marble (after Zhang et al., 2001).

permeability evolution is controlled by competition between rates of crack growth processes and rates of crack healing and sealing during deformation.

Experimental studies of microfracture healing (Brantley et al., 1990; Hickman and Evans, 1987; Zhang et al., 2001) demonstrate that, at temperatures greater than approximately $300^{\circ} \mathrm{C}$, diffusional crack healing and associated loss of crack connectivity in minerals such as quartz and calcite can be fast, even on laboratory time-scales (Fig. 2). Mineralsealed, grain-scale microfractures in exhumed mid-crustal shear zones similarly indicate that permeability is controlled by repeated growth and sealing of microfractures during ongoing deformation.

At high pore fluid factors, permeability in and around active shear zones is also provided by the growth of macroscopic extension fractures and shear fractures which are sealed by hydrothermal minerals to produce veins (Fig. 3(a)). The internal structure of veins in mid-crustal shear systems in some cases provides spectacular evidence for repeated fracturing and fracture-sealing during progressive deformation. For example, crack-seal microstructures in extension veins (Ramsay, 1980; Cox, 1995) indicate that vein formation can involve up to several thousand fracturing and sealing events (Fig. 3(b)).

A key result from the experimental studies, and from observations of natural fracture systems is that, because permeability is rapidly destroyed by mineral precipitation in fluidactive shear networks, fluid flow cannot be maintained unless ongoing deformation repeatedly regenerates crack connectivity.

\subsection{Fluid focussing in active shear zones}

The enhanced permeability of active shear systems relative to their host rocks perturbs crustal fluid flow patterns when shear zones connect with crustal fluid reservoirs. The significance of active shear zones in controlling fluid migration is illustrated by analytic solutions and numerical modelling studies of two-dimensional (2D) flow patterns around high permeability zones in a less permeable matrix (Phillips, 1991; Cox et al., 2001). For permeable structures inclined at 

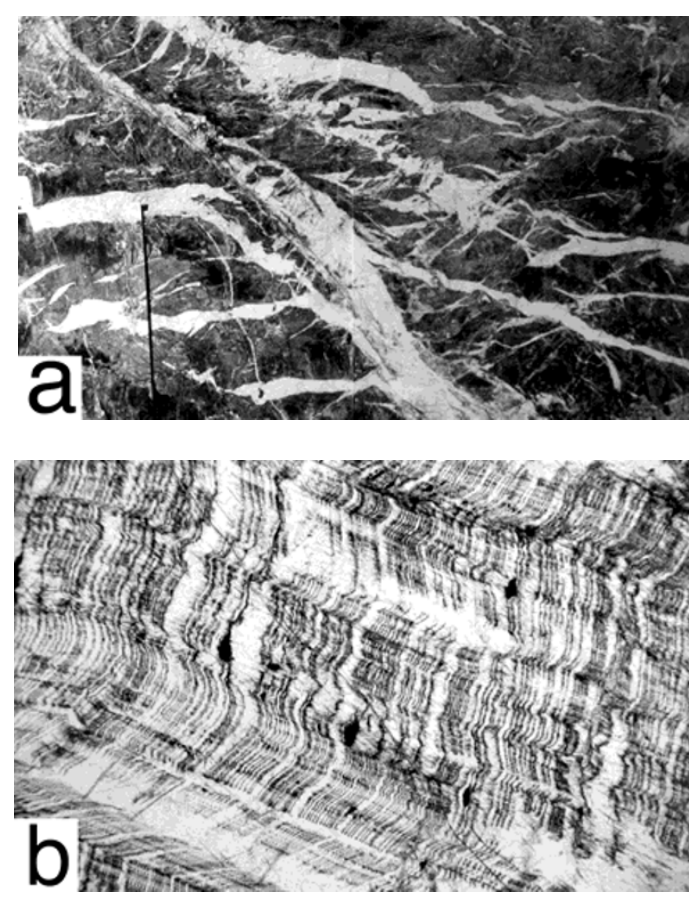

Fig. 3. (a) Arrays of externsion veins and fault-fill veins around a reverse shear zone record fluid pathways during shear zone development. Zone of light-coloured alteration in the metabasalt wall-rock adjacent to the veins record fluid infiltration from the fracture network into the wall-rock. Revenge Mine, St Ives goldfield, Western Australia. Field of view is 9 metres wide. (b) Quartz-rich extension vein with dark, crack-seal inclusion bands composed of tourmaline. This texture indicates multiple episodes of extensional fracturing and fracture-sealing associated with repeated slip on an adjacent reverse fault. Sigma mine, Val d'Or, Quebec. Field of view is $2 \mathrm{~mm}$ wide.

low angles to the regional gradient in hydraulic head, fluid focussing occurs around the higher pressure (upstream) levels of shear zones, whereas fluid discharge occurs in the lower pressure (downstream) levels of shear zones (Fig. 4). The dimensions of fluid charge regions are comparable to the lengths of the high permeability segments of faults and shear zones. Shear zones which are active at mid- to deep crustal levels, and connect with near-lithostatically pressured, deep fluid reservoirs (e.g. fluids generated by metamorphic devolatilisation) probably play a key role in focussing the migration of deeply-sourced fluids upwards to the base of the seismogenic regime in some geodynamic settings.

\subsection{Episodic versus continuous flow in shear systems}

Competition between porosity-creation processes and porosity-destruction processes in actively deforming rocks results in a contrast in styles of fluid flow between the upper crustal seismogenic regime and the lower crustal aseismic regime. At depths below the seismic-aseismic transition, where steady-state creep processes usually dominate, an approximate balance between rates of porositydestruction and porosity-creation in creeping shear zones can generate quasi-steady-state permeabilities that are higher than those of the surrounding, less rapidly deforming rock mass (Cox, 1999). This leads to essentially continuous fluid flow along actively creeping parts of shear networks. In contrast, at and above the seismic-aseismic transition, potentially large, cyclic changes in fault permeability are associated with episodic rupture and interseismic fracture sealing.

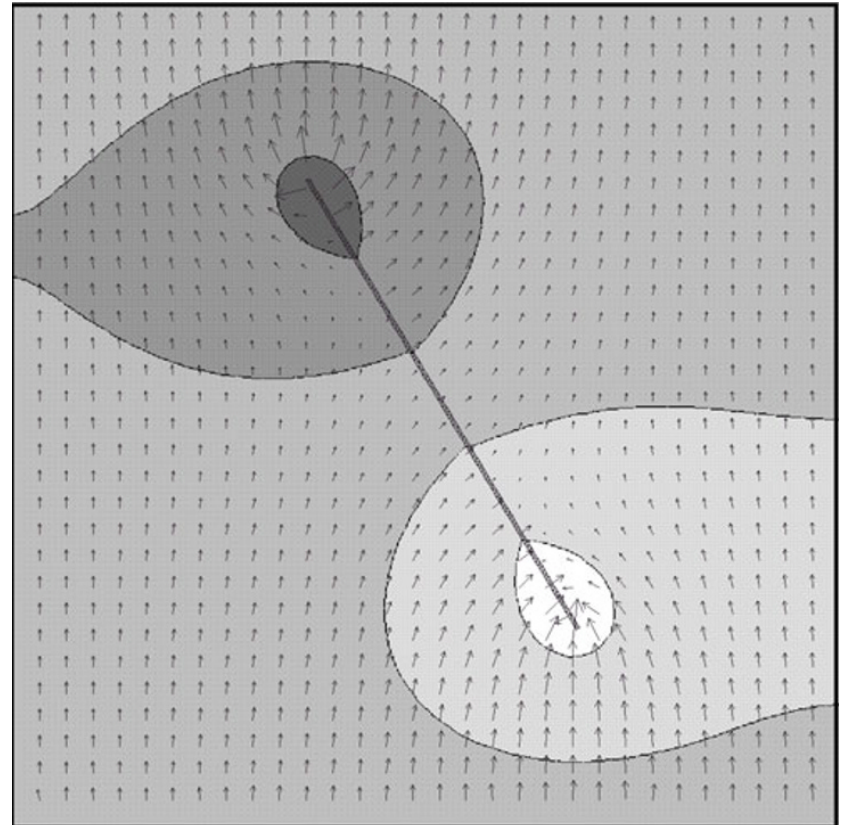

Fig. 4. Finite element model simulating steady-state, head-driven flow patterns around a permeable fault or shear zone embedded in a less permeable medium. A vertical lithostatic fluid pressure gradient is maintained in the medium away from the fault. Length of flow vectors corresponds to fluid flow velocity in the medium around the fault; flow vectors in the fault are not shown. Contours indicate departures of fluid pressure from lithostatic values; light areas are below and dark areas are above lithostatic pressure. Fault permeability is $10^{3}$ times that of the surrounding medium.

This leads to episodic fluid flow and fault-valve behaviour (Sibson, 1996; Cox, 1999; Miller and Nur, 2000). Episodic flow effects may transiently penetrate into the deeper crust during major fault slip events.

\section{Deformation Processes in Fluid-Active Crustal Shear Systems}

3.1 Structures associated with fluid-rock interaction in shear zones

Time-integrated fluid fluxes in shear zones exhumed from mid- to deep crustal levels can range up to $10^{6} \mathrm{~m}^{3} / \mathrm{m}^{2}$ (Ferry and Dipple, 1991). Evidence for fluid activity in shear zones includes the formation of macroscopic vein arrays, operation of dissolution-precipitation creep mechanisms, and hydrothermal alteration of shear zones during their formation. The extent of development of these structures is influenced by total fluid fluxes, pore fluid factors and rock reactivity.

Coupling between deformation processes and fluid flow in a high flux regime is spectacularly illustrated by structures associated with the development of mid-crustal shear systems which host gold deposits in the Archaean Yilgarn Craton of Western Australia. These shear systems have developed at about $2630 \mathrm{Ma}$ during strike-slip deformation in an openly folded sequence of upper greenschist facies metamorphosed basalts, gabbros and komatiites. Deformation occurred at depths of 10-12 km, at temperatures of approximately $400^{\circ} \mathrm{C}$, in a post-peak metamorphic regime. Most fluid flow was localised along networks of low displacement, reverse shear zones (Fig. 3(a)) that are spatially related to a major contractional jog in a crustal-scale, sinistral wrench 
shear system.

Displacement along the shear zones has involved both ductile and brittle behaviour. For example, at the Revenge mine, mineralised shears exhibit evidence for early brittle slip events and brecciation, followed by ductile shearing and development of shear zone foliations during progressive potassic alteration (Nguyen et al., 1998). Production of hydrothermal biotite, and associated reaction-weakening, during potassic alteration in the cores of shear zones has helped to localise ductile behaviour. Ductile shearing was punctuated by numerous, episodic brittle slip events which produced breccias and massive to laminated, quartz-rich shear veins, especially in jogs and dilatant bends in shear zones.

Intense sodic metasomatism and associated albite production in the core of shear zones, late in the displacement history, was associated with a transition to fully brittle behaviour. The widespread development of subhorizontal extension vein arrays adjacent to most shear zones indicates that the fluids migrating through them were at least episodically at supralithostatic pressures during much of the deformation history. Indeed, the distribution of high fluid fluxes and high pore fluid factors in the shear networks was probably a key factor driving growth of the shear zone networks and their propagation to higher crustal levels (Cox et al., 1987, 2001; Sibson, 1996).

\subsection{Influence of fluid pressure and shear stress on fail- ure modes}

First order transitions between brittle and plastic behaviour in crustal shear systems are commonly ascribed to the effects of increasing temperature and confining pressure (with increasing depth) which inhibit brittle processes and promote the operation of thermally-activated deformation processes such as dislocation creep and diffusional/advective mass transfer processes. However, the high fluid flux shear zones near Kambalda exhibit evidence for repeated transitions between brittle and plastic behaviour which cannot be ascribed to repeated fluctuations in temperature and depth. Deformation mode transitions are instead likely to be related to fluctuations in shear stress and fluid pressure associated with episodic fault slip and fault-valve behaviour in shear zones (Sibson et al., 1988).

The dependence of strength and failure modes in shear zones on episodic, fault-valve-driven, variations in shear stress and pore fluid pressures can be illustrated by failure mode diagrams which display brittle and plastic failure envelopes as a function of stress difference $\left(\sigma_{1}-\sigma_{3}\right)$ and pore fluid factor (fluid pressure/vertical stress) (Fig. 5).

Initial fluid flow in the shear systems near Kambalda was controlled by macroscopic shear fracture at pore factors near one, that is, at near-lithostatic fluid pressures (Fig. 5(a)). Reduced plastic shear strength, due to reaction-weakening associated with biotite production, progressively induced a transition to ductile deformation (Fig. 5(b)). In this deformation regime, fluid flow was controlled by permeability enhancement associated with grain-scale dilatancy and reaction-enhanced porosity, rather than macroscopic fracture permeability. Repeated brittle slip events, which occurred during ongoing ductile deformation, are interpreted to have been driven by increasing pore fluid factors during individual fault-valve cycles. Each brittle shear failure event was a

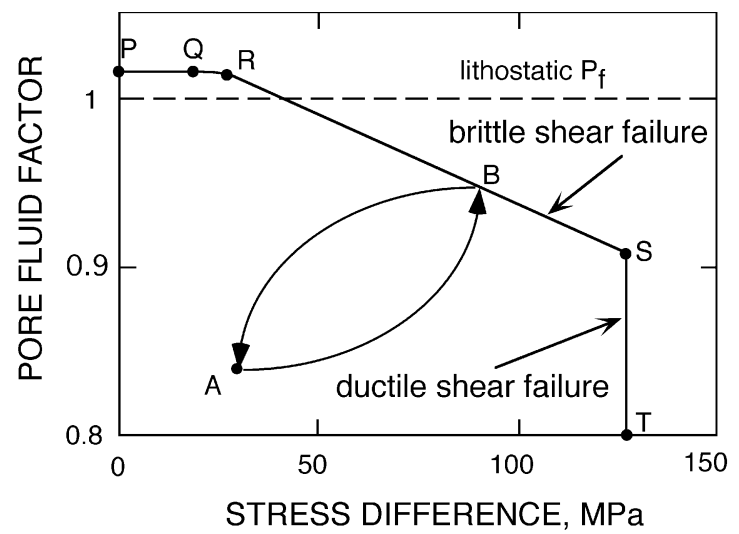

b

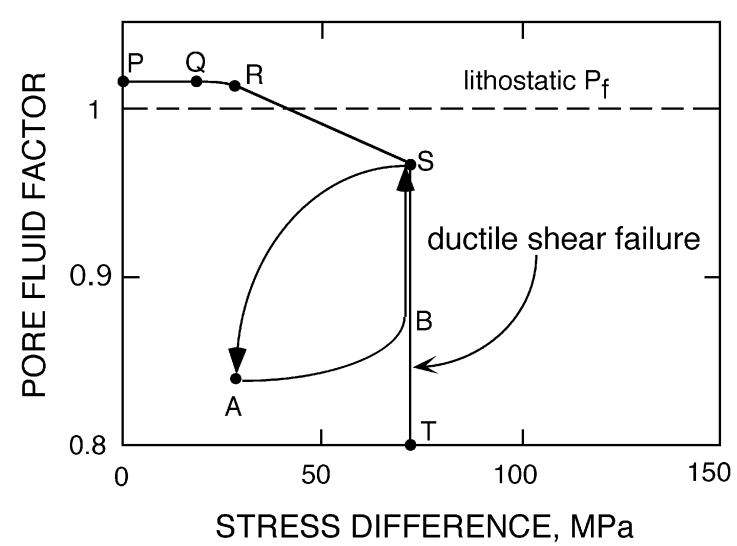

C

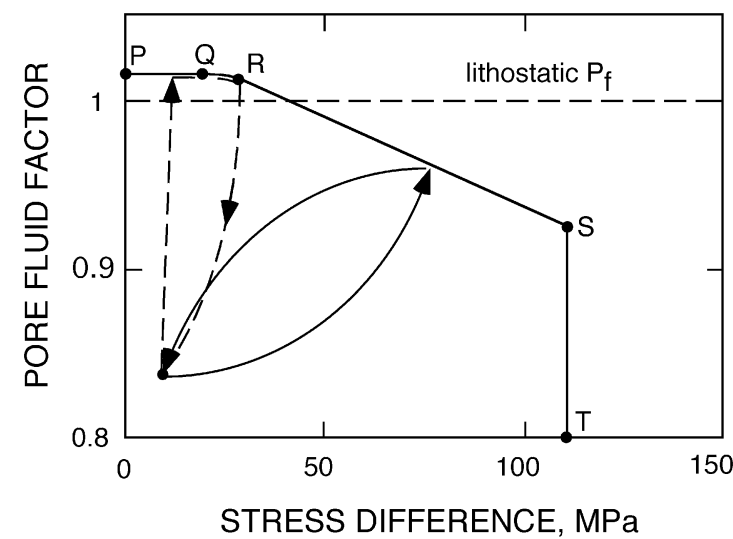

Fig. 5. Failure mode diagrams illustrating brittle and plastic failure envelopes as a function of pore fluid factor and stress difference. The diagram is constructed for optimally-oriented reverse faulting at a depth of $12 \mathrm{~km}$ in rock with a cohesive strength of $10 \mathrm{MPa}$, tensile strength of $5 \mathrm{MPa}$, and friction coefficient 0.75 . Fluid pressure and stress changes associated with fault-valve behavior may produce episodic transitions between no deformation, ductile shear failure, brittle shear failure, and brittle extension failure at various phases of the seismic cycle. The failure envelope is indicated by the curve PQRST. Ductile shear failure occurs for fluid pressure and stress states in the interval ST; brittle shear failure occurs between $\mathrm{S}$ and R; extensional-shear occurs between R and Q; and extensional failure occurs in the interval PQ. (a) Brittle shear failure at point B induced by increase in stress difference and fluid pressure. (b) Reaction-weakening decreases the ductile shear strength (ST), so that increase in stress difference and fluid pressure leads firstly to ductile shear failure (aseismic creep) at point B, followed by brittle shear failure at point $\mathrm{S}$, in response to progressive increase in pore fluid factor. (c) Late stage reaction-hardening increases ductile shear strength, so that failure occurs by brittle shear failure at high stress differences, or extension failure occurs at low stress differences and high pore fluid factors (after Cox et al., 2001). 
preceded by an interval of interseismic ductile shear at lower pore fluid factors (Fig. 5(b)). Sodic metasomatism, late in the hydrothermal history, produced localised albite-rich assemblages. This resulted in reaction-strengthening (plastic failure envelope moves to higher stress differences) and a transition back to fully brittle behaviour and macroscopic fracturecontrolled fluid flow late in the slip history (Fig. 5(c)). Note that extension failure occurs only if the rate of pore fluid factor increase is rapid relative to the rate of shear stress increase. Additionally, in a fault-valve scenario, hydraulic extension fracture can only occur prior to brittle slip events (point $\mathrm{R}$ in Fig. 5(c)). Lower rates of pore fluid factor increase, relative to shear stress increase, can induce brittle shear failure without prior extension failure. If pore fluid factors are less than about 0.9 , brittle shear failure is inhibited and aseismic creep will commence as soon as stress differences reach the plastic failure envelope (line ST in Fig. 5(c)).

The failure mode analysis highlights how the mechanical behaviour and shear strength of shear zones can be very dependent on pore fluid factors, shear stress and the nature of hydrothermal alteration reactions induced by migrating pore fluids. They also illustrate how transitions between aseismic creep, brittle shear failure and hydraulic extension failure are influenced by relative rates of change of pore fluid factors and shear stress during interseismic periods. A particularly important result is that even at constant shear stress, timedependent changes in pore factors alone may drive a shear system to failure.

Because of their enhanced permeability, actively creeping, mid- to deep crustal shear systems have a capacity to tap into various crustal fluid reservoirs. The four dimensional (4D) (i.e. space plus time) distribution of fluid flow during the evolution of these networks must play a critical role in supplying high pressure fluids to the base of the seismogenic regime, and therefore in driving the nucleation and recurrence of major earthquakes in the mid-crust. The 4D distribution of fluid flow is expected to be controlled by dynamically evolving connectivities amongst elements of shear networks and diverse fluid reservoirs. It will also be influenced by the distribution and geometry of the highest permeability sites, such as bends and jogs in major shear zones, and also junctions between shear zones.

Acknowledgments. This work was funded partly by a grant from the Australian Research Council, as well as by support from the ANU Research School of Earth Sciences and WMC Resources Ltd. S. Zhang, R. Sibson, J. Streit and P. Nguyen are thanked for discussion. J. Braun is thanked for numerical modelling. The photo for Fig. 3(a) was provided by P. Nguyen. I thank the organisers of the Sendai workshop for their generous support which allowed my participation. In particular, I thank K. Fujimoto and Y. Iio for review comments.

\section{References}

Brantley, S. L., B. Evans, S. H. Hickman, and D. A. Crerar, Healing of microcracks in quartz-implications for fluid flow, Geology, 18, 136139, 1990.

Cox, S. F., Faulting processes at high fluid pressures: an example of faultvalve behavior from the Wattle Gully Fault, Victoria, Australia, J. Geophys. Res., 100, 841-859, 1995.

Cox, S. F., Deformational controls on the dynamics of fluid flow in mesothermal gold systems, in Fractures, Fluid Flow and Mineralization, edited by K. McCaffrey, L. Lonergan, and J. Wilkinson, pp. 123-140, Geological Society, London, Special Publications, 155, 1999.

Cox, S. F., M. A. Etheridge, and V. J. Wall, The role of fluids in syntectonic mass transport, and the localization of metamorphic vein-type ore deposits, Ore Geology Reviews, 2, 65-86, 1987.

Cox, S. F., M. A. Knackstedt, and J. Braun, Principles of structural control on permeability and fluid flow in hydrothermal systems, Reviews in Economic Geology, 14, 1-24, 2001.

Ferry, J. M. and G. M. Dipple, Fluid flow, mineral reactions, and metasomatism, Geology, 19, 211-214, 1991.

Fischer, G. J. and M. S. Paterson, Measurements of permeability and storage capacity in rocks during deformation at high temperature and pressure, in Fault Mechanics and Transport Properties of Rocks, edited by B. Evans and T.-F. Wong, pp. 213-252, San Diego, Academic Press, 1992.

Hickman, S. H. and B. Evans, Influence of geometry upon crack healing in calcite, Physics and Chemistry of Minerals, 15, 91-102, 1987.

Holness, M. B., The permeability of non-deforming rock, in DeformationEnhanced Fluid Transport in the Earth's Crust and Mantle, edited by M. B. Holness, pp. 9-39, London, Chapman and Hall, 1997.

Miller, S. and A. Nur, Permeability as a toggle switch in fluid-controlled crustal processes, Earth Planet. Sci. Lett., 183, 133-146, 2000.

Nguyen, P. T., S. F. Cox, C. McA. Powell, and L. Harris, Fault-valve behaviour in optimally-oriented shear zones at Revenge gold mine, Kambalda, Western Australia, Journal of Structural Geology, 20, 1625-1640, 1998.

Peach, C. J. and C. J. Spiers, Influence of crystal plastic deformation on dilatancy and permeability development in synthetic salt rock, Tectonophys., 256, 101-128, 1996.

Phillips, O. M., Flow and Reactions in Permeable Rocks: Cambridge, U.K., 285 pp., Cambridge University Press, 1991.

Ramsay, J. G., The crack-seal mechanism of rock deformation, Nature, 284, 135-139, 1980.

Sibson, R. H., Structural permeability of fluid-driven fault-fracture meshes, Journal of Structural Geology, 18, 1031-1042, 1996.

Sibson, R. H., F. Robert, and K. H. Poulsen, High-angle reverse faults, fluidpressure cycling, and mesothermal gold deposits, Geology, 16, 551-555, 1988.

Stormont, J. C. and J. K. Daemen, Laboratory study of gas permeability changes in rock salt during deformation, International Journal of Rock Mechanics and Mining Science Geomechanics Abstracts, 29, 325-342, 1992.

Zhang, S., S. F. Cox, and M. S. Paterson, The influence of room temperature deformation on porosity and permeability in calcite aggregates, $J$. Geophys. Res., 99, 15761-15775, 1994.

Zhang, S., M. S. Paterson, and S. F. Cox, Microcrack growth and healing in deformed calcite aggregates, Tectonophys., 335, 17-36, 2001.

S. F. Cox (e-mail: sfcox@geology.anu.edu.au) 